\title{
Acute left main coronary artery occlusion following inadvertent delivery of radiofrequency energy during ventricular tachycardia ablation successfully treated by rescue angioplasty with stenting: A two-year follow-up
}

\author{
Piotr Waciński ${ }^{1}$, Andrzej Głowniak ${ }^{1}$, Elżbieta Czekajska-Chehab ${ }^{2}$, \\ Wojciech Dąbrowski ${ }^{3}$, Jarosław Wójcik ${ }^{1}$, Andrzej Wysokiński ${ }^{1}$ \\ ${ }^{1}$ Department of Cardiology, Medical University of Lublin, Lublin, Poland \\ ${ }^{2}$ Department of Radiology, Medical University of Lublin, Lublin, Poland \\ ${ }^{3}$ Department of Anesthesiology, Medical University of Lublin, Lublin, Poland
}

\begin{abstract}
Radiofrequency catheter ablation (RFCA) is a treatment mode in patients with recurrent, symptomatic, ventricular arrhythmias. A rare but potentially life-threatening complication of RFCA includes injury to the coronary arteries, which leads to acute occlusion and myocardial infarction. In the few reported cases, the most frequently affected vessel has been the left main coronary artery. We present the case of a 28 year-old female. During the RFCA procedure, an acute occlusion of the left main coronary artery occurred, which was treated successfully with emergency angioplasty. (Cardiol J 2013; 20, 1: 100-102)
\end{abstract}

Key words: radiofrequency catheter ablation, left main occlusion, coronary angioplasty

\section{Case report}

Our patient was a 28 year-old female with recurrent, drug-resistant ventricular arrhythmia, including non sustained ventricular tachycardia (nsVT) and ventricular ectopic beats (VEBs) ( $>10.000 / 24 \mathrm{~h}$ ).

Echocardiography revealed no structural heart disease. Given that the patient was symptomatic and the arrhythmia was resistant to drugs, she was referred to our center for radiofrequency catheter ablation (RFCA). Antiarrhythmic medication was discontinued $>5$ half-lives before the procedure. Following the initial activation and pace mapping in the right ventricular outflow tract, the mapping/ablation catheter (Marinr MC 4-mm tip, Medtronic, Fridley, MN, USA) was introduced into the left ven- tricle using a transaortic approach. The origin of the arrhythmia was localized in the left ventricular outflow tract, approximately $1.5 \mathrm{~cm}$ below the left aortic cusp (Fig. 1).

The mapping was supported by the electroanatomic system (LocaLisa, Medtronic) RF energy application $\left(50 \mathrm{~W} / 60^{\circ} \mathrm{C}\right)$ with a 4 -mm tip ablation catheter, resulting in suppression of the arrhythmia. After $43 \mathrm{~s}$ of RF application, the catheter suddenly dislocated, likely due to respiratory movement, and was moved above the aortic valve and into the ostium of the left main (LM) artery. The RF application was terminated immediately and the catheter was withdrawn. The patient developed severe symptoms of acute coronary hypoperfusion and cardiac arrest in the mechanism of asystole.

Address for correspondence: Piotr Waciński MD, Department of Cardiology, Medical University of Lublin, ul. Jaczewskiego 8, 20-950 Lublin, Poland, e-mail: piotr.wacinski@umlub.pl

Received: 31.12.2011

Accepted: 25.01.2012 


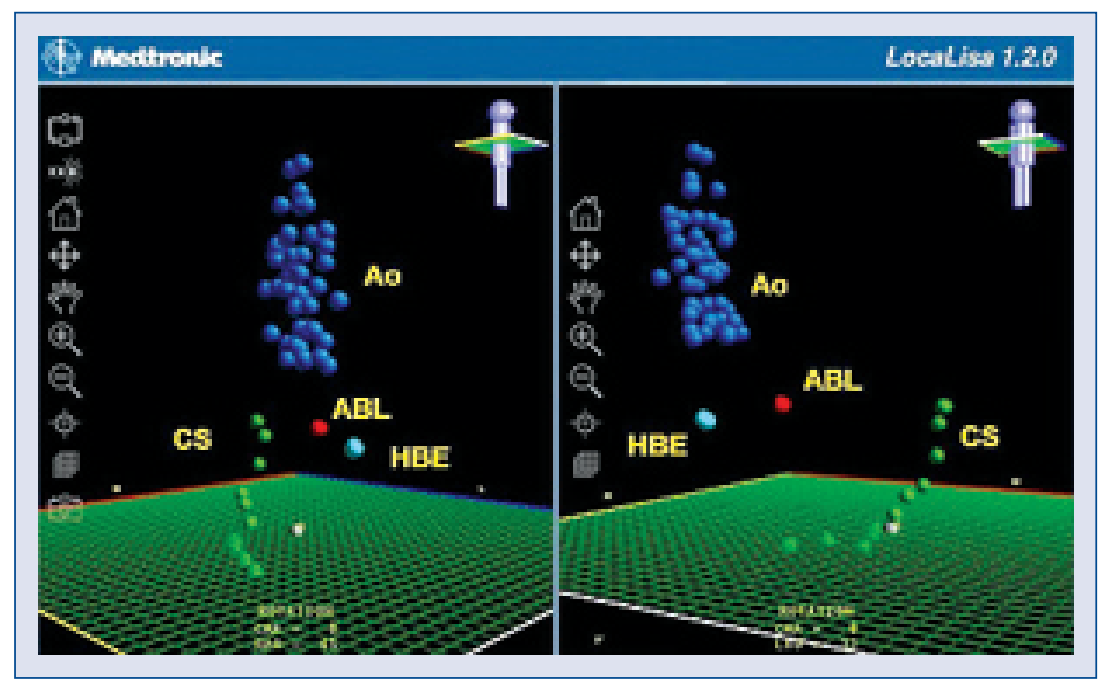

Figure 1. Position of the ablation site in the left ventricular outflow tract; $A B L$ - ablation site; CS — coronary sinus; HBE - His bundle electrogram; Ao - aortic root.

Immediate resuscitation resulted in the restoration of an idioventricular rhythm without sufficient hemodynamic response.

Echocardiography (in emergency) revealed important left-ventricle hypokinesis (antero-septal). With ongoing resuscitation, coronary angiography was performed, confirming acute occlusion of the LM artery. An electrode for intraventricular pacing was also inserted. In the first step, a direct stenting of the LM with a Cypher stent $(3.5 / 8 \mathrm{~mm}$; Cordis, Bridgewater, NJ, USA) was attempted. During stenting, a very strong contraction of the LM resulted in dislocation of the stent into the ostium of the LM. Stent thrombosis was observed. Treatment with abciximab (ReoPro, Eli Lilly, Indianapolis, IN, USA) was introduced, and the second stent (3.5/20 mm; CoCr stent, Skylor, Invatec, Roncadelle, Italy) was implanted from the LM to the left anterior descending, with TIMI 3 flow restoration. Hemodynamic heart function reappeared, with normal sinus rhythm. An intra-aortic balloon pump was inserted.

In the coronary care unit, the follow-up (FU) was complicated by pneumonia and heparin induced thrombocytopenia with the lowest platelet count at $32 \mathrm{~K} / \mu \mathrm{L}$.

A myocardial infarction was confirmed, with an increase of troponin I to a maximum of $>50.0 \mathrm{ng} /$ $/ \mathrm{mL}$. A control echocardiography revealed anteroseptal and apical hypokinesis with an ejection fraction of $45 \%$ on discharge.
In the second and third months after hospitalization, a control echocardiography was performed. The ejection fraction was calculated at $56 \%$, with only slight periapical dyskinesia.

After six months, the first control 64-multislice computed tomography was performed. Normal aspects for LM were observed, without restenosis. The ejection fraction was $51 \%$, and the left ventricular end-diastolic volume was $126 \mathrm{~mL}$ (Fig. 2).

Otherwise, the patient was asymptomatic. At the one-year FU, a computed tomography scan yielded similar results to those observed at the sixmonth FU (Fig. 2B). In the control ECG Holter, we identified no significant arrhythmias (VEBs 93/24 h, with no VT runs).

Currently, the patient is being treated with $25 \mathrm{mg}$ metoprolol and $75 \mathrm{mg}$ aspirin daily.

This case demonstrates a rare complication of RFCA $[1,2]$. The mechanism of the initial injury to the LM artery remains unclear; it is possible that the cause of the occlusion was an acute LM dissection. It should be evaluated whether precise delineation of the coronary anatomy before the application would increase the safety of the procedure.

These procedures are useful and in fact, mandatory when treating arrhythmias originating from aortic cusps, but not from sites below the aortic valve [3]. In this case, the coronary catheter placed in the LM could mechanically prevent the ablation catheter from entering into the vessel. Electro-anatomic mapping does not prevent the dislocation 


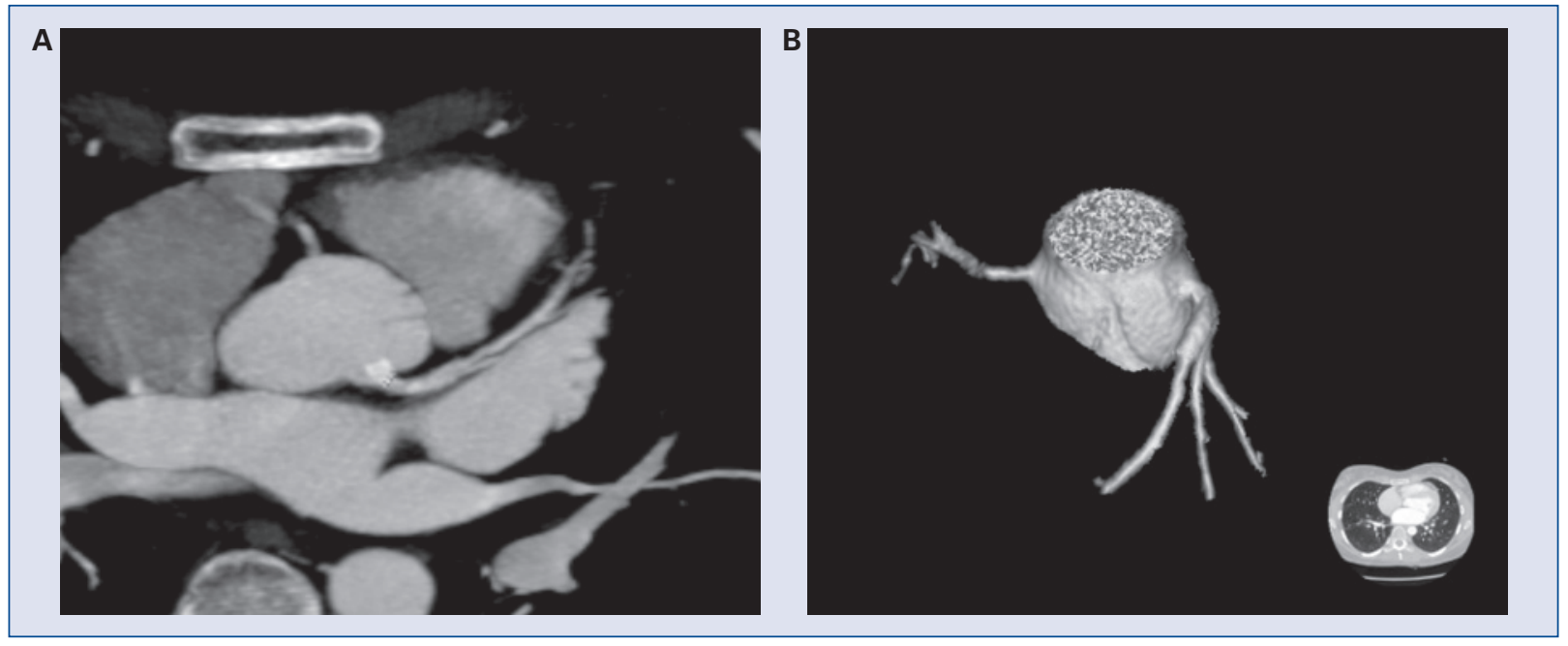

Figure 2. A. Multislice computed tomography scan showing the left main and left anterior descending/circumflex of the patient, without restenosis. The first stent slightly exteriorized into the left main ostium (six months follow-up); B. Normal aspect of the left coronary artery (one year follow-up).

of the ablation catheter. Thus, we recommend performing a coronary angiography (with the catheter left in the LM during RF application) not only in arrhythmias originating from the aortic cusps, but also for sub-valvular sites that are at risk of catheter dislocation.

Finally, little data exists regarding the long-term FU in patients with LM coronary artery stenting following an injury resulting from $\mathrm{RF}$ ablation.

Therefore, we intend to keep our patient under very close medical supervision for a long time.

Conflict of interest: none declared

\section{References}

1. Kharrat I, Charfeddine H, Sahnoun M et al. Left main coronary thrombosis: Unusual complication after radiofrequency ablation of left accessory atrioventricular pathway. J Electrocardiol, 2008; 41: 683-685.

2. Dinckal H, Yucel O, Kirilmaz A et al. Left anterior descending coronary artery occlusion after left lateral free wall accessory pathway ablation: What is the possible mechanism? Europace, 2003; 5: 263-266.

3. Aliot EM, Stevenson WG, Almendral-Garrote JM et al. EHRA/ /HRS Expert Consensus on Catheter Ablation of Ventricular Arrhythmias. Europace, 2009; 11: 771-817. 